\title{
Path Model of Mindfulness-Based Self-Efficacy on Rumination in Meditation Fields: Dual Mediating Effects of Self-Compassion and Dysfunctional Attitudes
}

\author{
Changhak Kyung ${ }^{1}$, HeeJung Lee ${ }^{2}$, and Sela Lim ${ }^{3 *}$ \\ ${ }^{1}$ Department of Clinical Psychology, NungIn University, Paltan-myeon, Hwaseong-si, \\ Gyeonggi-do, South Korea \\ ${ }^{2}$ Department of Naturopathic, Dongbang Culture University, Seongbuk-gu, Seoul, \\ South Korea \\ ${ }^{3}$ Department of Clinical Psychology, NungIn University, Paltan-myeon, Hwaseong-si, \\ Gyeonggi-do, South Korea \\ ${ }^{1}$ kchw6012@nate.com, 2 anahjlee57@naver.com, ${ }^{3 *}$ sera325@nate.com
}

\begin{abstract}
This study explored correlation of male and female citizen of Korea for Mindfulness-Based Self-Efficacy and Rumination to confirm and path of model Self-Compassion and Dysfunctional Attitudes. To this end, data was collected from 172 survey results conducted by 180. The survey consisted of, Mindfulness-Based Self-Efficacy Scale (MSES), Korean version of Self-Compassion Scale(K-SCS), Dysfunctional Attitudes Scale (DAS), Rumination Scale (RS). The results were analyzed by IBM SPSS V.22 and M-Plus 6.12 (Muthén \& Muthén, 1998-2011) program. To verify average and standard deviation of variables and mediation model of how Mindfulness-Based Self-Efficacy to Rumination through, Self-Compassion and Dysfunctional Attitudes the author used bootstrapping methods. For the research result, this study presented descriptive statics for each variable of respondents' and conducted correlation analysis among Mindfulness-Based Self-Efficacy to Self-Compassion $(\beta=.844$, $p<.001)$, and conducted correlation analysis among Self-Compassion to Dysfunctional Attitudes $(\beta=.725, \quad p<.001)$, and conducted correlation analysis among Dysfunctional Attitudes to Rumination $(\beta=.459, \quad p<.001)$. And Mindfulness-Based Self-Efficacy to Rumination through Self-Compassion and Dysfunctional Attitudes ( $\beta=.281$, C.I[.133 .548]) in due order.
\end{abstract}

Keywords: Mindfulness-based self-efficacy, Rumination, Path model

\section{Introduction}

Why do people meditate to have mindfulness-based self-efficacy and rumination? There seem to be basically two answers. First, people try to keep mindfulness-based self-efficacy because they want to overcome psychological or cognitional problems: mindfulness-based selfefficacy as a means for modification of dysfunctional attitudes [1]. Second, people meditate for mindfulness-based self-efficacy to achieve a better understanding of for self-compassion

Article history:

Received (February 16, 2020), Review Result (March 21, 2020), Accepted (April 30, 2020) 
for their life, enlarge their consciousness, and gain wisdom: mindfulness-based self-efficacy as a means to (positive) transformations in consciousness [2].

These two aims often cannot be clearly separated, and most practitioners of meditation probably pursue both to a certain extent.

People who have undergone extensive mindfulness-based self-efficacy training for mindfulness-based self-efficacy and Rumination have shown improvements on cognitive performance in Dysfunctional Attitudes and self-compassion. Despite the public's growing interest and an increasing number of studies on the impact of mindfulness-based self-efficacy and rumination, there is a surprising scarcity of summaries of the empirical evidence [3], especially for evidence that stems from research outside the therapeutic context within dysfunctional attitudes and self-compassion.

Numerous studies have been conducted to search for effects of meditation fields of study, yet there is an even more surprising lack of elaborate psychological theories that make sound predictions about what to expect if one meditates.

The traditional sense of meditation in Western culture, before significant encounter with Asian practices, involves sustained consideration or thought upon a subject. But at the moment Eastern studies culture using Western research method that psychological tools.

This a theoretical approach is frequently mirrored in the measures used in the studies, which include all kinds of dependent variables that are not specific to mindfulness-based selfefficacy and rumination research and have also been used in many other kinds of research [4].

Although, in this article, we place an emphasis on empirical evidence, we are convinced that real progress in understanding the effects of mindfulness-based self-efficacy and rumination cannot be made if future empirical studies are not guided by better theories [5].

Therefore, after briefly surveying previous attempts to summarize the literature on the effects of mindfulness-based self-efficacy, Rumination, Dysfunctional Attitudes and selfcompassion, we introduce existing theoretical approaches from the South Korea. Following our analysis of the empirical evidence, we return to the issue of how we might make progress in understanding mindfulness-based self-efficacy, rumination, dysfunctional attitudes and self-compassion and its effects.

There have long been attempts to bring together meditation for psychotherapy in South Korea [6], and over time, meditation has become an established psychotherapeutic technique [7]. There are even recently developed approaches to psychotherapy that center around meditation within mindfulness-based self-efficacy, rumination, self-compassion, dysfunctional attitudes and its effects. There have long been attempts to bring together meditation for psychotherapy [8], and over time, meditation has become an established psychotherapeutic technique [9].

There are even recently developed approaches to psychotherapy that center around meditation for mindfulness-based self-efficacy [10].

However, mindfulness-based self-efficacy and rumination perceived as positive cognition based on unconscious critical thoughts. It also causes psychological confusion and psychopathology. Kyung [11] reported the results of a positive correlation between dysfunctional attitudes and self-compassion.

Furthermore, comparative studies of research or cognition types focusing on psychological mindfulness have reported that psychological mindfulness positively affects victims' mental health as well as mindfulness-based self-efficacy and rumination and, in some cases, has more positive and long-term effects than dysfunctional attitudes and self-compassion [12].

As a result, it is found that there is a lack of research on mediating effects of mindfulnessbased self-efficacy, rumination, dysfunctional attitudes and self-compassion. 
Therefore, this study aims to investigate the relationship between mindfulness-based selfefficacy and rumination [13], and dysfunctional attitudes and self-compassion, and examines the mediating model between dysfunctional attitudes and self-compassion between mindfulness-based self-efficacy and rumination.

This study is based on the assumption that the issues of cognitional maladjustment due to various mindfulness-based self-efficacy and rumination experienced by meditator living of South Korea are related to dysfunctional attitudes and self-compassion.

It would be useful for enhancing the understanding of the factors affecting the meditator, and could provide the opportunity for the clinician to provide basic data on research and psychotherapy.

\section{Methods}

\subsection{Subjects and data collection}

This study was conducted from March 2018 to April 2018, for those who participated in the male and Female in Seoul of Korea. Finally, the data of total 172 participants were analyzed. [Table 1]

Table 1. General characteristics of subjects

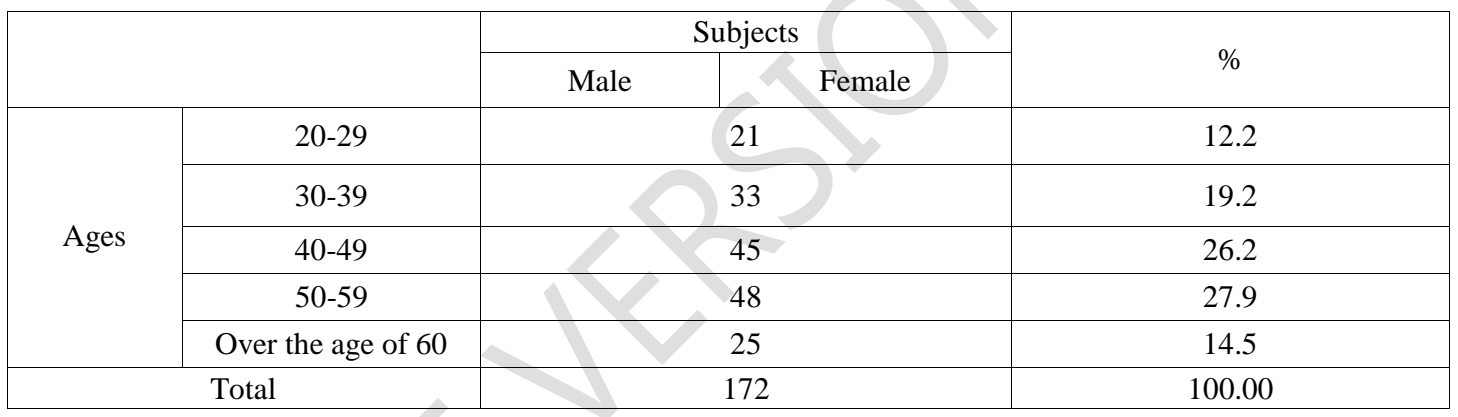

\subsection{Descriptive statistics of variable}

The Descriptive statistics of variable were analyzed [Table 2]

Table 2. Descriptive statistics of research variable

\begin{tabular}{|c|c|c|c|c|c|c|c|c|c|c|}
\hline \multirow{2}{*}{ Sex } & \multirow{2}{*}{ Age } & \multirow{2}{*}{$\mathrm{N}$} & \multicolumn{2}{|c|}{ Rumination } & \multicolumn{2}{c|}{$\begin{array}{c}\text { Dysfunctional } \\
\text { Attitudes }\end{array}$} & \multicolumn{2}{c|}{ Self-Compassion } & \multicolumn{2}{|c|}{$\begin{array}{c}\text { Mindfulness-Based Self- } \\
\text { Efficacy }\end{array}$} \\
\cline { 4 - 11 } & & $\mathrm{M}$ & $\mathrm{SD}$ & $\mathrm{M}$ & $\mathrm{SD}$ & $\mathrm{M}$ & $\mathrm{SD}$ & $\mathrm{M}$ & $\mathrm{SD}$ \\
\hline \multirow{4}{*}{ Male } & 3 & 5.08 & 0.00 & 4.77 & 0.00 & 4.85 & 0.00 & 4.54 & 0.00 \\
\hline & $30-39$ & 21 & 4.32 & 1.16 & 4.39 & 0.85 & 4.35 & 0.62 & 3.89 & 0.38 \\
\cline { 2 - 11 } & $40-49$ & 9 & 3.55 & 1.38 & 2.88 & 1.17 & 3.74 & 0.76 & 3.58 & 0.69 \\
\cline { 2 - 11 } & $50-59$ & 18 & 4.46 & 1.20 & 3.92 & 1.52 & 4.16 & 0.71 & 4.05 & 0.49 \\
\cline { 2 - 10 } & $\begin{array}{c}\text { Over the } \\
\text { age of } 60\end{array}$ & 15 & 3.61 & 1.63 & 3.60 & 1.28 & 3.97 & 0.82 & 3.88 & 0.60 \\
\cline { 2 - 10 } & Total & 66 & 4.12 & 1.34 & 3.89 & 1.28 & 4.15 & 0.73 & 3.92 & 0.53 \\
\hline Female & $20-29$ & 18 & 4.54 & 1.07 & 4.40 & 1.07 & 4.40 & 0.55 & 3.96 & 0.30 \\
\hline
\end{tabular}


Path Model of Mindfulness-Based Self-Efficacy on Rumination in Meditation Fields: Dual Mediating Effects of Self-Compassion and Dysfunctional Attitudes

\begin{tabular}{|c|c|c|c|c|c|c|c|c|c|c|}
\hline & $30-39$ & 12 & 4.92 & 0.24 & 4.69 & 1.01 & 4.64 & 0.28 & 4.29 & 0.30 \\
\hline & $40-49$ & 36 & 4.10 & 0.98 & 3.43 & 0.95 & 4.18 & 0.69 & 3.74 & 0.39 \\
\hline & $50-59$ & 30 & 3.66 & 1.37 & 2.88 & 1.29 & 3.93 & 0.69 & 3.69 & 0.41 \\
\hline & $\begin{array}{l}\text { Over the } \\
\text { age of } 60\end{array}$ & 10 & 3.71 & 1.33 & 2.41 & 0.83 & 3.29 & 0.34 & 3.37 & 0.35 \\
\hline & Total & 106 & 4.11 & 1.17 & 3.49 & 1.28 & 4.12 & 0.69 & 3.79 & 0.43 \\
\hline \multirow{6}{*}{ Total } & $20-29$ & 21 & 4.61 & 1.00 & 4.45 & 1.00 & 4.47 & 0.54 & 4.04 & 0.35 \\
\hline & $30-39$ & 33 & 4.53 & 0.98 & 4.50 & 0.91 & 4.45 & 0.54 & 4.04 & 0.40 \\
\hline & $40-49$ & 45 & 3.99 & 1.08 & 3.32 & 1.01 & 4.09 & 0.72 & 3.71 & 0.46 \\
\hline & $50-59$ & 48 & 3.96 & 1.35 & 3.27 & 1.46 & 4.02 & 0.70 & 3.83 & 0.47 \\
\hline & $\begin{array}{l}\text { Over the } \\
\text { age of } 60\end{array}$ & 25 & 3.65 & 1.49 & 3.12 & 1.25 & 3.70 & 0.74 & 3.68 & 0.57 \\
\hline & Total & 172 & 4.11 & 1.23 & 3.64 & 1.29 & 4.13 & 0.71 & 3.84 & 0.47 \\
\hline
\end{tabular}

Note: N(Number of people), M(Mean), SD(Standard deviation)

\subsection{Correlation analysis of variable}

The Correlation analysis of variable were analyzed. [Table 3]

Table 3. Correlation analysis of variable

\begin{tabular}{|c|c|c|c|c|}
\hline & Rumination & $\begin{array}{c}\text { Dysfunctional } \\
\text { Attitudes }\end{array}$ & Self-Compassion & $\begin{array}{c}\text { Mindfulness-Based Self- } \\
\text { Efficacy }\end{array}$ \\
\hline Rumination & 1 & & & \\
\hline $\begin{array}{c}\text { Dysfunctional } \\
\text { Attitudes }\end{array}$ & $.632 * *$ & 1 & 1 & \\
\hline Self-Compassion & $.571 * *$ & $.716^{* *}$ & $.844 * *$ & 1 \\
\hline $\begin{array}{c}\text { Mindfulness- } \\
\text { Based } \\
\text { Self-Efficacy }\end{array}$ & $.537 * *$ & $.601 * *$ & & \\
\hline
\end{tabular}

Note: $* p<.05 * * p<0.01 * * * p<.001$

\subsection{Measurement}

\subsubsection{Mindfulness-Based Self-Efficacy Scale (MSES)}

In order to measure mindfulness-based self-efficacy, we used the Mindfulness-Based SelfEfficacy Scale (MSES), which was developed by Cayoun and Freestun [14] which was developed for validation by Yong [2]. In the present study, the total of the mindfulness-based self-efficacy of Cronbach's $\alpha$ was .896 .

\subsubsection{Korean version of Self-Compassion Scale (K-SCS)}

In order to measure self-compassion, we used the Korean version of Self-Compassion Scale(K-SCS), which was developed by Neff [3]which was validated by Gu and Jung [15]. In the present study, the total of the self-compassion of Cronbach's $\alpha$ was .953. In this study, the sum of negative factor of self-compassion scale after the backscoring was used. 


\subsubsection{Dysfunctional Attitudes Scale (DAS)}

In order to measure dysfunctional attitudes, we used the Dysfunctional Attitudes Scale (DAS), which was developed by Jung and Oh and Shin [16]. In the present study, the total of the self-compassion of Cronbach's $\alpha$ was .979.

\subsubsection{Rumination Scale (RS)}

In order to measure dysfunctional attitudes, we used the Rumination Scale (RS), which was developed by Calhoun, Cann, Tedeschi and Mcmillan [17] which was translated by Shin [18] In the present study, the total of the self-compassion of Cronbach's $\alpha$ was .952.

\subsection{Data analysis}

The path model was used to test the mediating effect of self-compassion and Dysfunctional Attitudes in the relationship between Mindfulness-Based Self-Efficacy and Rumination. The model parameters were estimated by the maximum likelihood method, and M-Plus 6.12 [19] was used for the analysis. Data collected for this study were analyzed using the IBM SPSS V.22 as follows. We used a 95\% confidence interval based on the empirical distribution estimated by bootstrapping to verify the mediating effect. Bootstrapping interpreted the sample as statistically significant if it did not know the distribution of the population and if the $95 \%$ confidence interval did not include zero [20].

\section{Results}

\subsection{Path model}

A mindfulness-based self-efficacy analysis was conducted to confirm the effects of rumination. Specifically, the mindfulness-based self-efficacy and rumination has a significant effect on the self-compassion and dysfunctional attitudes, In addition, the mindfulness-based self-efficacy was found to have a significant effect on self-compassion $(\beta=.844, p<.001)$, and the self-compassion was found to have a significant dysfunctional attitudes $(\beta=.725, \mathrm{p}<.001)$, and the dysfunctional attitudes was found to have a significant rumination $(\beta=.459, \mathrm{p}<.001)$. A significant correlation was not found between mindfulness-based self-efficacy and dysfunctional attitudes, self-compassion and rumination, mindfulness-based self-efficacy and rumination [Table 2].

Table 2. Path model

\begin{tabular}{|c|c|c|c|c|c|c|c|}
\hline \multicolumn{2}{|c|}{ Path } & B & B & s.e & t & p \\
\hline \multirow{2}{*}{$\begin{array}{c}\text { Mindfulness- } \\
\text { Based Self- } \\
\text { Efficacy }\end{array}$} & $\rightarrow$ & Self-Compassion & 1.255 & .844 & .022 & 38.414 & .000 \\
\cline { 2 - 8 } & $\rightarrow$ & $\begin{array}{c}\text { Dysfunctional } \\
\text { Attitudes }\end{array}$ & -0.03 & -.011 & .099 & -0.113 & .910 \\
\cline { 2 - 8 } & Rumination & 0.508 & .196 & .106 & 1.850 & .064 \\
\hline $\begin{array}{c}\text { Self- } \\
\text { Compassion }\end{array}$ & $\rightarrow$ & $\begin{array}{c}\text { Dysfunctional } \\
\text { Attitudes }\end{array}$ & 1.325 & .725 & .091 & 7.931 & .000 \\
\cline { 2 - 8 } & \begin{tabular}{c} 
Rumination \\
\cline { 2 - 7 }
\end{tabular} & 0.134 & .077 & .122 & 0.633 & .527 \\
\hline
\end{tabular}




\begin{tabular}{|c|c|c|c|c|c|c|c|}
\hline $\begin{array}{c}\text { Dysfunctional } \\
\text { Attitudes }\end{array}$ & $\rightarrow$ & Rumination & 0.438 & .459 & .078 & 5.898 & .000 \\
\hline \multicolumn{7}{|c|}{$* \mathrm{p}<.05 * * \mathrm{p}<0.01 * * * \mathrm{p}<.001$} \\
\hline
\end{tabular}

\subsection{Measurement model}

We performed bootstrapping by extracting 2000 parts of the escapism of mindfulnessbased self-efficacy to the rumination through the dysfunctional attitudes and self-compassion. As a result, the indirect effect was significant because the path through the dysfunctional attitudes and self-compassion included 0 in the $95 \%$ confidence interval of the indirect effect $(\beta=.281$, C.I[.133 .548]) [Table 2].

Table 2. Measurement model

\begin{tabular}{|c|c|c|c|c|c|c|c|}
\hline $\begin{array}{c}\text { Independent } \\
\text { variable }\end{array}$ & Parameter 1 & Parameter2 & $\begin{array}{c}\text { Dependent } \\
\text { variable }\end{array}$ & $\begin{array}{c}\text { Total effect } \\
\text { [bootstrapping } \\
\text { CI 95\%] }\end{array}$ & $\begin{array}{c}\text { Direct effect } \\
\text { [bootstrapping } \\
\text { CI } 95 \%]\end{array}$ & $\begin{array}{c}\text { Total indirect } \\
\text { effect } \\
{[\text { bootstrapping }} \\
\text { CI 95\%] }\end{array}$ & $\begin{array}{c}\text { Indirect effect } \\
\text { [bootstrapping CI } \\
95 \% \text { ] }\end{array}$ \\
\hline $\begin{array}{l}\text { Mindfulness- } \\
\text { Based Self- } \\
\text { Efficacy }\end{array}$ & $\begin{array}{c}\text { Self- } \\
\text { Compassion }\end{array}$ & $\begin{array}{l}\text { Dysfunctiona } \\
\text { Attitudes }\end{array}$ & Rumination & & & & $.281[.133 \sim .548]$ \\
\hline $\begin{array}{c}\text { Mindfulness- } \\
\text { Based Self- } \\
\text { Efficacy }\end{array}$ & $\begin{array}{c}\text { Self- } \\
\text { Compassion }\end{array}$ & 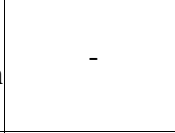 & Rumination & $.537[.405$ & & $\begin{array}{c}.340 \\
{[.133 \sim .548]}\end{array}$ & $.065[-.181 \sim .296]$ \\
\hline $\begin{array}{c}\text { Mindfulness- } \\
\text { Based Self- } \\
\text { Efficacy }\end{array}$ & - & $\begin{array}{c}\text { Dysfunctiona } \\
\text { Attitudes }\end{array}$ & $\mathrm{Ru}$ & & & & $\begin{array}{c}-.005[-.090 \\
\sim .082]\end{array}$ \\
\hline $\begin{array}{l}\text { Mindfulness- } \\
\text { Based Self- } \\
\text { Efficacy }\end{array}$ & & & Rumination & & $\begin{array}{c}.196[- \\
.057 \sim .436]\end{array}$ & - & - \\
\hline
\end{tabular}

\section{Conclusions}

The results of this study suggest that mindfulness-based self-efficacy of meditation experienced people has a significant effect on rumination through dysfunctional attitudes and self-compassion.

Meanwhile, a simple correlation analysis, revealed that there was a significant correlation between mindfulness-based self-efficacy, rumination, dysfunctional attitudes and selfcompassion, whereas the path search for the median model analysis was significant.

This is consistent with a previous study [1], which reported a significant correlation between mindfulness-based self-efficacy, of meditation experienced people and dysfunctional attitudes. Lee [1] discovered that most causes of mindfulness-based self-efficacy that interfere with dysfunctional attitudes and self-compassion after meditation, is experienced in daily life.

Choi [21] discovered among meditation experienced people that most of the causes of mindfulness-based self-efficacy that interfere with thinking are related to self-compassion, individual cognitional healthy lifestyle and experiences of rumination in daily life.

Kang [22] discovered among meditation experienced people that most of the causes of mindfulness that interfere with cognitional healthy school life and deliberate rumination are 
related to self-Disclosure and posttraumatic growth responses, individual lifestyle, role, and experiences in daily school life.

Gilbert [23] reported that self-compassion is an important factor that affects rumination. Yun [24] reported a significant correlation between self-compassion with dysfunctional attitudes.

Gwak [25] discovered the effectiveness of Thought Control Strategies on Rumination.

Therefore, mindfulness-based self-efficacy of changes in these cognition conditions could lead to a deterioration of psychological function.

What we have outlined above can be seen as a continuum that appears at this point to have returned to its beginning. We start with the workings of the ordinary mind, which takes anything that happens to appear in the mind or senses as an object of awareness, but in an undisciplined and apparently random way. According to Buddhist thought, nothing is truly random in the human mind.

however, what appears to be the spontaneously attentive mind is actually a mind reacting to phenomena with host of unconscious cognition and attitudes that mindfulness-based selfefficacy and dysfunctional attitudes and self-compassion and rumination. When, in mindfulness-based self-efficacy, awareness is encouraged to roam freely over the phenomena of experience, it does so with qualitatively more clarity and continuity than is accessible in ordinary states of mind.

In conclusion, the mediating effect of mindfulness-based self-efficacy on rumination and the dysfunctional attitudes and self-compassion of meditation experienced people was solely analyzed in South Korea, and significant results were obtained.

Nevertheless, it is important to note that results from this study could be used in reference to cognitional mental health especially during counseling interventions and in clinical settings.

\section{References}

[1] H. Y. Lee, "The effects of mindfulness meditation on the maximization and dysfunctional attitude of maximizes," M.S. thesis, Department of Psychology Graduate School, Duksung Women's University, South Korea, (2009)

[2] H. C. Yong and K. Y, Hwang, "The relation of dysfunctional meta-cognition, meta-mood, and gibchak," East West Mind Science, vol.9, no.2, pp.15-31, (2006) DOI:10.1080/15298860309027

[3] K. D. Neff, "The development and validation of a scale to measure self-compassion," Self and Identity, vol.2, no.3, pp.223-250, (2003) DOI:10.1080/15298860390209035

[4] J. E. Ha, "The effect of the adult attachment anxiety on posttraumatic stress and growth: mediating effect of self-compassion and brooding," Ph.D. dissertation, Department of Education in Guidance and Counseling, Graduate School of Sookmyung Women's University, South Korea, (2003)

[5] S. R. Park, "The effect of self-compassion on self-regulation: The effectiveness of the self-compassion enhancement program," Ph.D. dissertation, Department of Psychology, Graduate School, Seoul National University, South Korea, (2015)

[6] C. K. Germer, "The mindful path to self-compassion," The Guilford Press, New York, pp.260, (2009)

[7] R. G. Tedeschi and L. G. Calhoun, "Posttraumatic growth: conceptual foundations and empirical evidence," Psychological Inquiry, vol.15, pp.1-18, (2004) DOI:10.1207/s15327965pli1501_01

[8] Y. J. Park, "The effect of intrusive rumination, deliberate rumination and self-compassion on post-traumatic growth," M.S. thesis, Department of counseling, Korea Counseling, Graduate University, South Korea, (2018)

[9] L. G. Calhoun and R. G. Thdeschi, "Facilitating posttraumatic growth; A clinician's guide," Lawrence Erlbaum Associates, Mahwah, New Jersey, pp.130, (1999) 
[10] M. E. Francis and J. W. Pennebaker, "The impact of writing on physiological, absentee, and self-reported emotional well-being measures," American Journal of Health Promotion, vol.6, no.4, pp.280-287, (1992) DOI:10.4278/0890-1171-6.4.280

[11] C. H. Kyung, "Effects of mindfulness-based self-efficacy on rumination: dual mediating effects of selfcompassion and dysfunctional attitudes," M.S. thesis, Department of Meditation Psychology, NungIn Graduate School of University, (2019)

[12] D. A. Taylor, R. J. Gould, and P. J. Brounstein, "Effects of personality self-disclosure," Personality and Social Psychology Bulletin, vol.7, no.3, pp.487-492, (1981) DOI:10.1177/014616728173019

[13] E. J. Ko, "Analysis on the emotional effect of self-compassion and self-esteem in the occurrence of negative life events," Ph.D. dissertation Department of psychology, Graduate School, Korea University, (2014)

[14] B. A. Cayoun and J. Freestun, "Mindfulness-based self-efficacy," Psych Technologies, vol.30, no.2, (2004)

[15] D. Y. Gu and M. C. Jung, "Study of factor validity of Korean version self-compassion," Journal of Korea Contents Association, vol.16, no.9, pp.160-169, (2016)

[16] M. I. Jung, S. S. Oh, and H. K. shin, "The attribution of success: failure experience in functional and dysfunctional perfectionists,” Korean Journal of Clinical Psychology, vol.24, no.3, pp.709-719, (2005)

[17] L. G. Calhoun, A. Cann, R. G, Tedeschi, and J. Mcmillan, "A correlation test of the relationship between posttraumatic growth, religion and cognitive processing” Journal of Traumatic Stress, vol.13, pp.21-527, (2000) DOI:10.1023/a:1007745627077

[18] S. Y. Shin, "The effect of meaning in life and social support on posttraumatic growth: rumination as a mediating variable," M.S. thesis, Department of counseling, Graduate School, Catholic University, (2009)

[19] L. K. Muthén and B. O. Muthén, “Mplus; Statistical analysis with latent variables user's guide,” Los Angeles, California, pp.78, (2012)

[20] P. E. Shrout and N. Bolger, "Mediation in experimental and no experimental studies; new procedures and recommendations," Psychological Methods, vol.7, no.4, pp.422, (2002) DOI:10.1037/1082-989x.7.4.422

[21] B. Y. Choi, "Mindfulness to gratitude and forgiveness: mediation effects by self-compassion and compassion for other," M.S. thesis, Department of Psychology the Graduate School Elwha Women's University, (2017)

[22] H. J. Kang, "Influence of self-disclosure on posttraumatic growth: dual mediating effects of mindfulness and deliberate rumination," M.S. thesis, Department of Counseling, Graduate School, Catholic University, (2019)

[23] P. Gilbert and S. Proctor, "Compassionate mind training for people with high shame and self-criticism: overview and pilot study of a group therapy approach," Clinical Psychology and Psychotherapy, vol.13, no.6, pp.353-379, (2006) DOI:10.1002/cpp.507

[24] J. Y. Yoon, "The influences of maladaptive perfectionism on anger expression styles: the mediating effects of emotional clarity and self-compassion," M.S. thesis, Department of counseling, Graduate School, Catholic University, (2018)

[25] A. R. Gwak, "The mediating effects of thought control strategies on the relationship between event-related rumination and posttraumatic growth," M.S. thesis, Department of counseling, Graduate School, Catholic University, (2015) 